ARTIGO DE REVISÃO

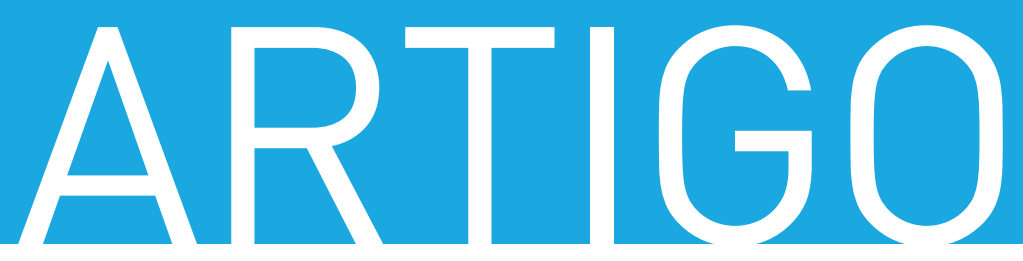

\title{
PROTOCOLOS PARA TRATAMENTO PSICOLÓGICO EM PANDEMIAS: AS DEMANDAS EM SAUDE MENTAL PRODUZIDAS PELA COVID-19
}

\author{
PROTOCOLS FOR PSYCHOLOGICAL INTERVENTIONS IN \\ PANDEMICS: THE MENTAL HEALTH DEMANDS CAUSED BY \\ COVID-19
}

\section{Resumo}

O presente trabalho discute e sugere estudos de desenvolvimento de protocolos para atendimento em saúde mental frente à situação de emergência e crise proporcionada pela COVID-19. Há evidências de repercussões na saúde mental e restrições no modo de enfrentamento da pandemia, tendo em vista a situação de isolamento e confinamento prolongadas pela quarentena, sejam entre os suspeitos de contágio, os enfermos pela COVID-19, os familiares ou as equipes de saúde envolvidas na linha de frente do tratamento. Entretanto, há escassez de literatura técnico-científica de protocolos de atendimento para pessoas com necessidade de afastamento social e do trabalho em função da pandemia.

Palavras-chave: Saúde mental, COVID-19, pandemia, protocolo, impactos psicológicos.

\section{Abstract}

This paper discusses and suggests studies dealing with development of protocols for mental health interventions in the emergency and crisis situation caused by COVID-19. There is evidence of repercussions for mental health and limitations on the means for dealing with the pandemic, caused by the prolonged isolation and confinement imposed by quarantine measures, observable in contagion suspects, COVID-9 patients, family members, and members of the healthcare teams involved in frontline treatment. However, there is scant technical and scientific literature on intervention protocols for people obliged to endure withdrawal from social and employment activity because of the pandemic.

Keywords: Mental health, COVID-19, protocols, pandemic, psychological impacts.

No final de 2019, um novo surto de pneumonia se espalhou na população mundial, causado por um vírus denominado síndrome respiratória aguda grave - coronavírus 2 (SARS-CoV-2), também conhecido como COVID-19, originado na China, o que obrigou os diferentes países a se mobilizarem para enfrentar as consequências da contaminação na saúde e na economia. A quarentena, forma principal de contenção da velocidade da contaminação pela COVID-19 e da letalidade, adotada em várias regiões do mundo, promoveu o isolamento e o confinamento de um grande número de pessoas e a mobilização de um contingente significativo de profissionais da saúde para o enfrentamento da situação de crise $^{1}$. A dimensão global dos reflexos da COVID-19 fez com que a Organização Mundial de Saúde (OMS) reconhecesse a sua transmissão como uma pandemia ${ }^{2,3}$.

Apesar das medidas adotadas para conter a propagação da COVID-19, governos, profissionais e instalações hospitalares não se mostraram preparados para mitigar os seus efeitos ${ }^{1,2,4}$. Uma das principais medidas adotadas pelos países afetados foi a quarentena, que é a separação e a restrição da movimentação de pessoas que tenham sido potencialmente expostas ao contágio de uma determinada doença, com o objetivo de reduzir 
o risco de infectarem outras pessoas. A quarentena é considerada um recurso extremo, mas eficaz, contra a disseminação do vírus e diminuição de novos casos, sobretudo entre os principais grupos de risco: idosos, indivíduos com comorbidades respiratórias e cardíacas 5 .

Ficar em quarentena é diferente de ficar em isolamento, situação na qual os indivíduos já infectados e confirmados para determinada doença são isolados para evitar o contágio. Durante uma pandemia, a quarentena é recomendada, porém pode ser vivenciada como uma experiência pessoal desagradável, desconfortável ou dolorosa, inclusive para os familiares. Há separação e ao mesmo tempo confinamento de famílias, rituais de luto são suspendidos, há sentimento de perda da liberdade, solidão, tédio, incertezas quanto ao futuro, suicídios, assim como ataques de pânico e raivab .

A humanidade já passou por epidemias como a síndrome respiratória aguda grave (SARS), Zika, ebola, H1N1 e, certamente, enfrentará novas epidemias devido à disseminação de doenças infecciosas com a globalização. A pandemia provocada pela COVID-19 tem produzido aumento de sintomas de ansiedade e pressões psicológicas de uma maneira geral na população. O estresse psicológico associado às situações de emergências e crises não pode ser ignorado. O cenário produzido pela COVID-19 é percebido como uma oportunidade para o desenvolvimento de estratégias de orientação, atenção e tratamento à saúde mental dos envolvidos ${ }^{2,7-9}$.

Dados clínicos e epidemiológicos sobre as implicações da COVID-19 na saúde mental ainda são recentes. Em um estudo sobre os impactos psicológicos gerados pela pandemia do novo coronavírus na China ${ }^{4}$, em um intervalo de 14 dias (entre janeiro e fevereiro de 2020), foram coletados dados acerca das reações a eventos estressores e percepção de sintomas estresse, ansiedade e depressão em 1.210 pessoas de 194 cidades chinesas, por meio das escalas Event Scale - Revised (IES-R) e Depression, Anxiety and Stress Scale (DASS-21). Os resultados mostram que o impacto dessa epidemia foi considerado de moderado a grave para mais de 50\% dos respondentes, sendo que $75 \%$ das pessoas estavam "muito preocupadas" ou "preocupadas" sobre as suas famílias serem contaminadas pela COVID-19. Dos participantes dessa pesquisa, 46,5\% expressaram alto nível de confiança no médico para diagnosticar a COVID-
19, e os que não confiavam nos médicos apresentaram escores significativos na subescala de estresse, ansiedade e depressão da DASS-21. Por sua vez, pacientes que referiram baixa probabilidade de contrair a COVID-19 apresentavam baixo escore na subescala de estresse da DASS-21, ao contrário dos pacientes que referiram maior probabilidade de contrair a COVID-19.

Os pacientes infectados pela COVID-19, os familiares desses pacientes, as equipes de saúde e outras pessoas afetadas por essa pandemia precisam de suporte psicológico, mas essa população está sendo pouco amparada ${ }^{2}$. A China, por meio de diretrizes formuladas pela sua Comissão Nacional de Saúde [National Health Commission of China (NHCC)], apresentou um guia para intervenções em pacientes com a COVID-19. Cabe aos países afetados pela pandemia aperfeiçoar o modelo apresentado pela NHCC, se for o caso, ou construir protocolos mais afetos às características das suas respectivas populações, cultura e modus operandi do trabalho de seus profissionais de saúde.

Em uma pandemia, a exacerbação de sinais e sintomas de transtornos mentais e a inabilidade para o enfrentamento de situações de emergência são esperadas. Em um certo sentido, elas agem como obstáculos às boas práticas de atenção e tratamento especializado e podem comprometer a melhoria do quadro clínico dos pacientes infectados. Há uma situação de tensão social que faz com que se promova o isolamento de milhões de pessoas em suas residências, para tentar controlar a exposição ao vírus e, ao mesmo tempo, o deslocamento de equipes de saúde e equipamentos para a linha de frente, aumentando o risco de contaminação biológica e letalidade nesses profissionais. A equipe médica, mesmo estando em contato direto com o vírus, pontua menos nos inventários de trauma do que a população em geral; entretanto, apresentam outros gatilhos para o estresse, como perturbações nas redes familiares, necessidade de adaptação cultural, quando se trata de equipe médica internacional, ansiedade, temor de contaminação pela alimentação e trabalho excessivo $0^{7,9}$. Esses profissionais precisam, por isso, se proteger da fadiga e tensão excessivas, da exaustão resultante da recusa ao descanso e da necessidade de manter a concentração e atenção em alerta para não se contaminarem. Embora as medidas apontem resultados menos significativos entre as equipes 


\section{ARTIGO DE REVISÃO}

GRAZIELE ZWIELEWSKI

GABRIELA OLTRAMARI

ADAIR ROBERTO SOARES SANTOS

EMANUELLA MELINA DA SILVA NICOLAZZI

JOSIANE ALBANÁS DE MOURA

VÂNIA L. P. SANT'ANA

RACHEL SCHLINDWEIN-ZANINI

ROBERTO MORAES CRUZ
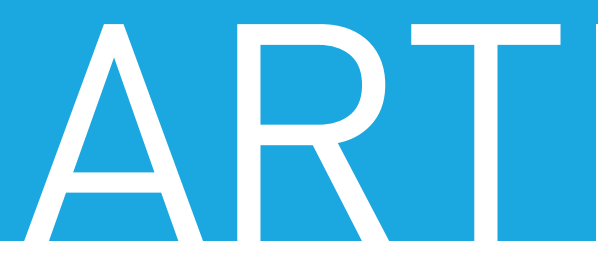

GO médicas quando comparados com aqueles obtidos junto à população em geral, é necessário implementar meios para a redução do estresse, ansiedade, insônia, tristeza, mágoa, desamparo, depressão, frustração e culpa diante da morte de pacientes.

Entre os grupos envolvidos com a COVID-19, é possível destacar os pacientes com o diagnóstico da doença, a equipe médica, os familiares e os suspeitos. Os infectados pela COVID-19 podem apresentar diversos sintomas psicológicos, os quais vão variar conforme os estágios de tratamento da doença, desde o diagnóstico até o isolamento hospitalar ou doméstico ${ }^{10}$. Os pacientes podem sentir raiva, medo, ansiedade, insônia, estresse e apresentar risco de suicídio e comportamentos de autolesão?. Todos esses sintomas podem prejudicar o tratamento durante o processo de adoecimento e perdurar por um tempo maior após o retorno ao período de normalidade de movimentação ${ }^{1}$. Da mesma maneira que as equipes médicas, os pacientes suspeitos que apresentam sintomas semelhantes aos provocados pela contaminação da COVID-19 também vivenciam sentimentos de pânico durante o período de isolamento e espera pelo resultado da confirmação da doença, que nem sempre é disponibilizado para o paciente, a não ser que o caso seja grave ${ }^{11}$.

Não apenas os suspeitos, mas os pacientes e as equipes médicas sofrem com os efeitos de uma pandemia. A população, em geral, também apresenta maior pontuação nos inventários de rastreio de sintomas de trauma, quando comparada com os resultados obtidos pela equipe médica ${ }^{8}$. Nesse caso, observa-se que a pandemia atinge outras esferas da vida além da saúde: o fechamento de escolas e a nova rotina com as crianças, o fechamento de empresas e dos locais públicos, as mudanças na dinâmica de trabalho e na reorganização familiar. A população apresenta reações de quem está sendo invadido por sentimento de impotência, insegurança, desamparo e pelo medo do futuro, já que a vida das pessoas está em risco e a economia dos países está incerta.

A Tabela 1 apresenta, de forma sintética, as demandas específicas dos grupos envolvidos na pandemia da COVID-19.

Tabela 1 - Demandas específicas dos grupos envolvidos na pandemia da COVID-19

\section{Grupo envolvido com a COVID-19} Demandas específicas de cada grupo identificadas na literatura especializada

Suspeitos As intervenções precisam ser baseadas na psicoeducação sobre a doença, observação cuidadosa dos sintomas, aderência ao tratamento precoce, adoção de medidas de proteção necessárias, relato aos órgãos de saúde, trabalhar para redução do estresse.

Famílias Os familiares, além do estresse do isolamento, precisam lidar com a incerteza e o medo de perder o ente querido que está com COVID-19 e, em alguns casos, lidar com o luto.

Profissionais das equipes da linha de frente

\section{Pressão psicológica insuportável: ansiedade, medo, depressão, insônia.}

Reduzir a enorme pressão, incluindo o alto risco de infecção devido à exposição e à inadequada proteção contra a contaminação, excesso de trabalho, frustração, discriminação, isolamento ou redução do contato com seus familiares.

Reduzir a pressão sobre os riscos inerentes ao trabalho, tais como a alta probabilidade de contrair uma infecção devido à exposição continuada e à inadequada proteção contra a contaminação, excesso de trabalho, frustração, discriminação, isolamento ou redução do contato com seus familiares.

Plataforma online com aconselhamento médico para pacientes e para a população em geral, onde são fornecidas informações sobre o risco de transmissão, reduzindo assim a pressão em cima da equipe de saúde.

Entre os temas que precisam ser abordados pelos protocolos com os profissionais de saúde, estão: manejo do estresse, trauma, depressão, comportamentos de risco, psicoeducação, monitoramento e controle da raiva e agressividade, desenvolvimento de sentimentos de pertencimento, assim como o incentivo à prática de atividades físicas.
Fonte(s) de informação

$\mathrm{NHCC}^{12}$

$\mathrm{CDC}^{13}$

$\mathrm{CDC}^{13}$

$\mathrm{NHCC}^{12}$

Handbook of COVID-19 Prevention and Treatment ${ }^{14}$

Li et al. ${ }^{8}$ 


\section{COVID-19}

GRAZIELE ZWIELEWSKID, GABRIELA OLTRAMARID, ADAIR ROBERTO SOARES SANTOS E, EMANUELLA MELINA DA SILVA NICOLAZZIC, JOSIANE ALBANÁS DE MOURAD, VÂNIA L. P. SANT'ANA RACHEL SCHLINDWEIN-ZANINID, ROBERTO MORAES CRUZ

Universidade Federal de Santa Catarina (UFSC), Florianópolis, SC.

Tabela 1 - Demandas específicas dos grupos envolvidos na pandemia da COVID-19

\section{Grupo envolvido com a COVID-19}

Pacientes

\section{Demandas específicas de cada grupo identificadas na literatura especializada}

As pessoas que apresentam a confirmação da doença sentem medo sobre a sua condição de enfermidade e referem temor pelo que pode acontecer consigo mesmas e com os seus familiares. Podem apresentar mudanças no sono ou no padrão alimentar, além de dificuldades para se concentrar. Nessa condição, pode haver o agravamento dos seus problemas crônicos de saúde, além do uso de álcool, tabaco e outras drogas.

O estado mental dos pacientes deve ser monitorado semanalmente, após a admissão e antes da alta, considerando o humor, a qualidade do sono e o estresse, por meio das ferramentas sugeridas pelo Handbook of COVID-19 Prevention and Treatment (2020): Self-Reporting Questionnaire 20 (SRQ-20), Patient Health Questionnaire 9 (PHQ-9), Generalized Anxiety Disorder 7 (GAD-7), Hamilton Depression Rating Scale (HAM-D), Hamilton Anxiety Rating Scale (HAM-A) e Positive and Negative Syndrome Scale (PANSS).

Durante o estágio inicial do tratamento, o paciente costuma negar a seriedade e as consequências da doença, assim como sentir raiva, medo, ansiedade, insônia. As diretrizes da NHCC descrevem que, nesse período, sejam compreendidas como normais essas respostas emocionais ao estresse, inclusive precisando o profissional estar preparado para o comportamento agressivo do paciente. Deve ser investigado o risco de suicídio, autolesão, apoio familiar. Nesse primeiro momento, é necessário explicar a importância do isolamento e ajudar o paciente a ter expectativas de uma recuperação positiva.

Durante o período de isolamento, é normal que o paciente tenha dificuldades com sono, o sentimento de solidão aumentado, medo devido aos riscos da doença, culpa pela possibilidade de ter contaminado outras pessoas. Alguns pacientes demonstram vontade de abandonar o tratamento, enquanto outros têm expectativas irrealistas e altas a respeito do tratamento ${ }^{2-12}$. Para esse período, é importante explicar sobre a doença e a situação externa, auxiliar na comunicação com parentes, incentivar que o paciente coopere com o tratamento e instruir que o paciente receba ajuda psiquiátrica, se necessário.

Para pacientes com dificuldade respiratória, ansiedade extrema e dificuldade de se expressar, é sugerido que se trabalhe com técnicas para acalmar o paciente e regular as emoções, assim como para melhorar a confiança no tratamento.

No caso de pacientes leves em isolamento domiciliar, as diretrizes da NHCC ${ }^{12}$ são de que se trabalhe para reduzir sentimentos de pânico, inquietação e raiva, ajudar os pacientes a compreenderem a ressignificar sentimentos de desamparo, vergonha ou desconsideração pelo seu estado de saúde, dificuldades com o sono.

É importante ajudar os pacientes a filtrarem informações reais e confiáveis, como materiais científicos e médicos, e não se afetarem pelas chamadas fake news, assim como incentivar a prática da leitura e da escuta de músicas. Esses pacientes também precisam ser ajudados na aceitação do isolamento, na aceitação de que a comunicação para redução da solidão será exclusivamente por meios de comunicação e nunca de forma presencial; precisam ser incentivados a manter contato com amigos por meio desses canais de comunicação e a buscarem apoio psicológico online.

Para pacientes com sintomas leves, é sugerido fazer o treino de respiração, relaxamento e atenção plena. Já para pacientes de moderado a grave, a indicação é que o tratamento seja combinado com medicação: novos antidepressivos, ansiolíticos e benzodiazepínicos podem ser administrados para melhorar o humor e a qualidade do sono. Quetiapina ou olanzapina podem ser recomendados para controlar sintomas psicóticos.

Os pacientes precisam se distrair ao invés de procurarem leituras e notícias sobre a doença. É necessário que sejam orientados a fazer pausas ao ler, assistir ou ouvir notícias. Precisam manter os cuidados com o corpo e a mente: exercícios físicos, alongamentos, técnicas de respiração e meditações. Sugere-se, ainda, refeições saudáveis e equilibradas, bem como um sono de qualidade e momentos de relaxamento. Por fim, que sejam incentivados a se conectar com pessoas, falar de suas preocupações e de como estão se sentindo.
Fonte(s) de informação

$\mathrm{NHCC}^{12}$

Handbook of COVID-19

Prevention and Treatment ${ }^{14}$

Xiang et al. ${ }^{2}$

\section{$\mathrm{CDC}^{13}$}

População

Medo, pânico generalizado, estigmatização e exclusão de pessoas confirmadas e sobreviventes, reações 
A China, primeiro país a enfrentar a pandemia, registrou o maior declínio na sua produção industrial desde os anos 90 e teve queda nas suas exportações, atingindo a cadeia produtiva de indústrias de muitos países. $O$ isolamento e o decreto de algumas províncias chinesas para o exercício do período de quarentena fez com que o comércio fosse fechado por vários dias e afetasse a economia local, a lucratividade e as responsabilidades financeiras das empresas ${ }^{4-6}$. No Brasil, assim como em outros contextos internacionais, o medo pelo desemprego, o excesso de informações e fake news, a solidão do isolamento, a inatividade e os conflitos entre familiares devido à convivência intensa no período de isolamento elevam o nível do estresse da população, obrigada a conviver com restrições severas na movimentação e rotina das pessoas ${ }^{1}$.

Estudos relacionados às consequências para a saúde mental e as medidas tomadas, em 2002, durante a epidemia da SARS sugerem que medidas semelhantes poderiam ser utilizadas como fontes de informações para equipes criarem protocolos de intervenções em saúde mental ${ }^{2}$. Durante a SARS, foram utilizadas linhas telefônicas para aconselhamento psicológico. No entanto, não ocorreu uma organização e gerenciamento das intervenções psicológicas, o que fez com que os profissionais da área da saúde mental atendessem a demanda sem se comunicarem uns com os outros, o que gerou um diagnóstico insatisfatório e um atendimento sem acompanhamento adequado ${ }^{7}$. Essa experiência sugere a necessidade de desenvolvimento de um protocolo de intervenção, de modo a proporcionar maior assertividade nos atendimentos em saúde mental.

Os efeitos psicológicos em pessoas que estavam em quarentena podem ser comparados àqueles verificados em pessoas que não estavam em quarentena em situações similares ao do novo coronavírus ${ }^{6}$. Profissionais de saúde que cuidaram de pacientes em quarentena estavam mais propensos a apresentarem exaustão e ansiedade, além de irritabilidade, insônia, baixa concentração, indecisão e deterioração do desempenho no trabalho. O efeito de estar em quarentena foi considerado um fator de pré-disposição para sintomas de estresse pós-traumático, mesmo 3 anos após o surto. Também foram encontrados sintomas de depressão na população sob quarentena, sendo $9 \%$ de sintomas de depressão grave. Relatos de uso abusivo de álcool e comportamentos dependentes também foram evidenciados, assim como o comportamento evitativo, apesar do fim da quarentena. O estudo ${ }^{6}$ mostra que uma pandemia traz consequências psicológicas para a população, mesmo ao término do evento de contaminação do vírus, o que reforça a necessidade de protocolos de intervenção psicológica para pandemias com necessidade de isolamento, como a de COVID-19.

\section{PROTOCOLOS PARA ATENDIMENTO EM SITUAÇõES DE EMERGÊNCIA E PANDEMIAS}

Protocolos, de uma maneira geral, podem ser definidos como um conjunto de orientações ou diretrizes sobre como realizar uma intervenção profissional. No âmbito da saúde, protocolo são diretrizes, instruções e/ou procedimentos específicos a serem seguidos pelos especialistas para conduzir um processo de investigação ou intervenção com pacientes, com o objetivo de orientar decisões e sobre como utilizar critérios técnicoscientíficos?. Protocolos para atendimento e cuidados na saúde de populações são úteis para padronizar e otimizar processos de triagem, rastreio, diagnóstico, tratamento medicamentoso ou psicoterapêutico ${ }^{16} \mathrm{em}$ pacientes e demais pessoas envolvidas em situações de emergência ou crises, como é o caso nesse momento.

Pode-se inferir que a população em geral carece de amparo psicológico ao se deparar com estados excepcionais de eventos estressores, tais como desastres e pandemias. Além do temor à contaminação experimentado pela população, as demandas por atenção e cuidados clínicos em termos de saúde mental são manifestadas por suspeitos de contaminação, familiares, profissionais de saúde da linha de frente e pelos pacientes. Para cada um desses grupos, são indicados protocolos breves de atendimento e focados na demanda ou queixa, visando dispor de procedimentos mais eficazes de contenção ou remissão de sintomas e inabilidades para o enfrentamento de estressores. A terapia cognitivo-comportamental (TCC) tem sido a estratégia clínica de tratamento psicológico mais recomendada para a consecução desses objetivos.

As TCC partem de pressupostos cognitivos para explicar as reações psicológicas e os padrões comportamentais relacionados aos sintomas: a) a avaliação de si mesmo, dos outros e das consequências das suas decisões; b) as 


\section{COVID-19}

GRAZIELE ZWIELEWSKID, GABRIELA OLTRAMARID, ADAIR ROBERTO SOARES SANTOSO, EMANUELLA MELINA DA SILVA NICOLAZZI JOSIANE ALBANÁS DE MOURA@, VÂNIA L. P. SANT'ANA RACHEL SCHLINDWEIN-ZANINID, ROBERTO MORAES CRUZC

Universidade Federal de Santa Catarina (UFSC), Florianópolis, SC

crenças e padrões emocionais funcionais e disfuncionais relacionadas desencadeadas frente às situações estressoras; c) os erros de julgamento e decisão para promover o enfrentamento de problemas ${ }^{11}$. De fato, há evidências de que intervenções psicológicas baseadas na TCC, inclusive aquelas realizadas online ou por telefone ${ }^{17}$, podem contribuir na compreensão do processo de proliferação de doenças dessa magnitude de exposição e suas repercussões na saúde e no comportamento pessoal e dos familiares. Nessas situações, os pacientes e familiares tendem a superestimar os riscos, mostram-se ansiosos, com medo e apresentam dificuldade para lidar com os estressores ambientais e com o isolamento.

Psicólogos e psiquiatras também precisam estar na linha de frente das equipes de saúde que cuidam dos envolvidos na COVID-19, e para isso é necessário desenvolver formas de atendimento emergencial às demandas em saúde mental. Na prática, serviços de emergência psiquiátricos e plantões psicológicos constituem espaços de escuta, acolhimento e mitigação de sintomas e, apesar de recorrerem a metodologias de atendimento típicas, não são baseados em protocolos específicos de atendimento.

$\mathrm{Na}$ literatura especializada acerca de situações de emergências e pandemias, não há informações concretas sobre modelos de protocolos de atendimento completos em saúde mental, capazes de avaliar a demanda pelo serviço, bem como especificar, por meio de procedimentos sistemáticos e estruturados de intervenção, as etapas do acolhimento, de rastreio de sintomas pré e pós-intervenção, as atividades de psicoeducação, as estratégias de enfrentamento e de resolução de problemas ${ }^{4,7}$. No caso da COVID-19, há contribuições importantes sobre a identificação de impactos das pandemias na saúde mental da população e sugestões de intervenções psicológicas para melhorar a saúde mental e a resiliência psicológica de pacientes e profissionais da saúde 1,3,4,7.

A população em geral, que sofre os desconfortos da quarentena, necessita de informações de boa qualidade sobre como reagir e enfrentar os problemas advindos com a situação de emergência; ou seja, promover processos educativos sobre o risco de contágio, práticas de higiene e demais medidas de segurança mais recomendadas para o enfrentamento decorrentes da pandemia ${ }^{1,7}$. E esses processos são fundamentais para o controle da crença nas falsas notícias, da ansiedade e do descontrole emocional. Por tudo isso, é importante promover programas de psicoeducação ao longo e após o período de quarentena para mitigar os impactos psicológicos do isolamento e confinamento infligidos, sob pena de se instalarem estigmas sociais, processos de rejeição e de afastamento das pessoas entre si e do convívio nas comunidades.

Entre os instrumentos utilizados para a psicoeducação, é sugerida a elaboração de uma checklist com todos os passos importantes para a prevenção da transmissão a ser fornecida à toda população sujeita ao contágio, para que ela possa consultar e ter a certeza de que está fazendo o necessário para evitá-lo4 . A percepção positiva acerca das medidas de precaução e prevenção à proliferação da doença leva a um efeito positivo nas respostas psicológicas. Além de protocolos de atendimento em saúde mental, enfatiza-se a importância de as autoridades oferecerem aplicativos ou canais exclusivos para as atualizações e comunicações sobre as características e os impactos das pandemias, de modo a evitar a disseminação de notícias falsas ${ }^{1,4}$. Há relatos de maior grau de satisfação com as informações recebidas associado com menor impacto psicológico da pandemia e níveis mais baixos de estresse, ansiedade e depressão $0^{2,7}$.

\section{ConCLUSÃo}

Um investimento em protocolos de atendimento que atinjam o maior número possível de pessoas sob o estresse de uma pandemia traria resultados positivos no que se refere à diminuição da ansiedade, do medo e demais sensações e sentimentos desagradáveis causados pela quarentena e pelo isolamento ${ }^{15,18}$. Estamos diante de uma pandemia com grande impacto social e de dimensão internacional, em que as intervenções tradicionais para os cuidados com a saúde mental não se adequam às necessidades atuais. $\bigcirc$ que tínhamos, até então, eram situações de emergências e desastres naturais em que o contato do psicólogo com as pessoas afetadas era realizada de modo presencial e permitia a prestação dos primeiros atendimentos psicológicos (PAP) recomendados pela OMS1.

É necessário, porém, o desenvolvimento de novos modelos de intervenção em saúde mental para serem 


\section{ARTIGO DE REVISÃO}

GRAZIELE ZWIELEWSKI

GABRIELA OLTRAMARI

ADAIR ROBERTO SOARES SANTOS

EMANUELLA MELINA DA SILVA NICOLAZZI

JOSIANE ALBANÁS DE MOURA

VÂNIA L. P. SANT'ANA

RACHEL SCHLINDWEIN-ZANINI

ROBERTO MORAES CRUZ
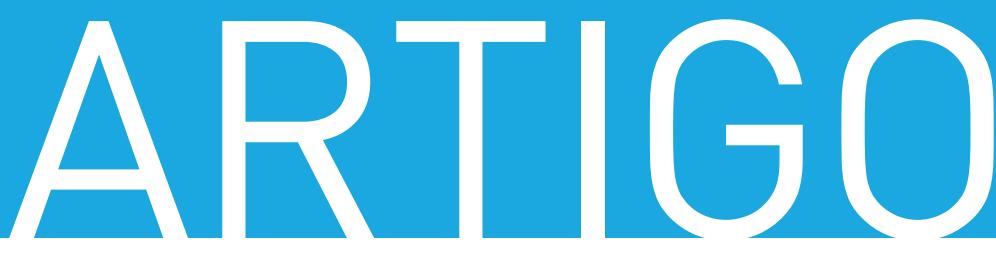

disponibilizados à população; intervenções que pressuponham novas políticas públicas e treinamentos para as equipes de suporte à saúde mental, assim como protocolos que induzam intervenções mais eficazes para o tratamento psiquiátrico e psicológico ${ }^{2-7}$. Atendimentos online precisam ser facilitados, permitindo o uso de técnicas e instrumentos informatizados para rastrear características da demanda, histórico clínico e sintomas de transtornos mentais entre os atingidos pelos impactos da pandemia. Aplicativos que possibilitem, de forma remota, gerenciar consultas e administrar testes psicológicos que contribuam favoravelmente no atendimento dos problemas em saúde mental e também nas pesquisas futuras sobre o tratamento psicológico dos grupos afetados pela quarentena e isolamentos prolongados.

Para tanto, é necessária a criação de protocolos de intervenção psicológica úteis ao tratamento dos problemas de saúde mental, que auxiliam na melhoria do humor dos pacientes, na remissão de sintomas, que busquem a resolução de problemas demandados e que possam promover a psicoeducação dos processos de aprendizagem voltados ao enfrentamento de estressores provocados pela situação de pandemia. Os procedimentos e protocolos atuais, voltados a situações de emergência e desastres, tendem a se restringir a atendimentos presenciais ${ }^{1}$.

Parece haver consenso entre os especialistas ${ }^{2-6}$ sobre o que precisa ser feito para auxiliar os quatro grupos envolvidos na pandemia da COVID-19: suspeitos, família, equipes e infectados, mas em nenhum dos referenciais teóricos pesquisados foi evidenciada uma metodologia específica sobre como se devem fazer essas intervenções. Há sugestões de procedimentos psicoterapêuticos baseados na terapia cognitiva, porém sem uma descrição estruturada ou protocolo para a sua realização ${ }^{4}$.

A TCC parece ser uma opção de intervenção eficaz para ser realizada durante ou imediatamente após a vivência de situações de crise social e pandemias, em que são adotadas condições de isolamento e restrições acentuadas de movimentação. Além disso, a TCC permite criar um plano de tratamento psicológico breve e adequado às demandas dos diferentes grupos populacionais envolvidos nessas situações.
Por fim, é importante refletir sobre os desafios da validação e padronização de protocolos de atendimento ou tratamento psicológico a serem desenvolvidos após as pesquisas resultantes das experiências da pandemia da COVID-19. A comparação entre os resultados obtidos no uso de protocolos nas modalidades presencial e online serão inevitáveis. Este artigo procurou discutir alguns aspectos que sinalizem os desafios na organização e implementação de protocolos para tratamento de problemas de saúde mental em situação de emergências e pandemias. A expectativa é contar com a proliferação de estudos que subsidiem a padronização desses protocolos num futuro próximo.

Artigo submetido em 05/04/2020, aceito em 06/04/2020. Os autores informam não haver conflitos de interesse associados à publicação deste artigo.

Fontes de financiamento inexistentes.

Correspondência: Roberto Moraes Cruz, Rua Natalícia Pereira, 129, João Paulo, 88030-361, Florianópolis, SC. Email: robertocruzdr@gmail.com

\section{Referências}

1. Barros-Delben P, Cruz RM, Trevisan KRR, Gai MJP, Carvalho RVC, Carlotto PAC et al. Saúde mental em situação de emergência: Covid-19. Debates Psiquiatr. 2020; [Epub ahead of print]

2. Xiang YT, Yang Y, Li W, Zhang L, Zhang Q, Cheung $\mathrm{T}$, et al. Timely mental health care for the 2019 novel coronavirus outbreak is urgently needed. Lancet Psychiatry. 2020;7:228-9.

3. Ornell F, Schuch JB, Sordi AO, Kessler F. "Pandemic fear" and COVID-19: mental health burden and strategies. Braz J Psychiatry. 2020 Apr 3. pii: S1516-44462020005008201. doi: 10.1590/1516-4446-2020-0008. [Epub ahead of print]

4. Wang C, Pan R, Wan X, Tan Y, Xu L, Ho CS, et al. Immediate psychological responses and associated factors during the initial stage of the 2019 Coronavirus Disease (COVID-19) epidemic among the General Population in China. Int J Environ Res Public Health. 2020 Mar 6;17(5). pii: E1729. doi: 10.3390/ijerph17051729. 
Universidade Federal de Santa Catarina (UFSC), Florianópolis, SC.

5. Lin EC, Peng YC, Tsai JC. Lessons learned from the anti-SARS quarantine experience in a hospitalbased fever screening station in Taiwan. Am J Infect Control. 2010;38:302-7.

6. Brooks SK, Webster RK, Smith LE, Woodland L, Wessely S, Greenberg N, et al. The psychological impact of quarantine and how to reduce it: rapid review of the evidence. Lancet. 2020;39:912-20.

7. Duan L, Zhu G. Psychological interventions for people affected by the COVID-19 epidemic. Lancet Psychiatry. 2020;7:300-2.

8. Li W, Yang Y, Liu ZH, Zhao YJ, Zhang Q, Zhang L, et al. Progression of mental health services during the covid-19 outbreak in China. Int J Biol Sci. 2020;16:1732-8.

9. Heymann T. Clinical protocols are key to quality health care delivery. Int J Health Care Qual Assur. 1994;7:14-7.

10. Kang L, Li Y, Hu S, Chen M, Yang C, Yang BX, et al. The mental health of medical workers in Whuhan, China dealing with the 2019 novel coronavirus. Lancet Psychiatry. 2020;7:e14.

11. Naeem F, Irfan M, Javed A. Coping with COVID19: urgent need for building resilience through cognitive behaviour therapy. Khyber Med University J. 2020;12:1-3.

12. National Health Commission of China (NHCC). A notice on the issuance of guidelines for emergency psychological crisis intervention in pneumonia for novel coronavirus infections [Internet]. 2020 [cited 2020 Mar 21]. www.nhc.gov.cn/xcs/zheng cwj/202001/6adc08b966594253b2b791be5c3b 9467.shtml
13. Centers for Disease Control and Prevention (CDC). Mental health and coping during COVID19 [Internet]. 2020 [cited 2020 Mar 26]. www.cdc. gov/coronavirus/2019-ncov/daily-life-coping/ managing-stress-anxiety.html?

14. Handbook of Covid-19 prevention and treatment: compiled according to clinical experience [Internet]. 2020 [cited 2020 Mar 22]. https:// gmcc.alibabadoctor.com/prevention-manual/ reader?pdf=Handbook\%20of\%20COVID-19\%20 Prevention\%20and\%20Treatment\%20(Standard). pdf\&opt=read\&version=standard\&language $=e n \&$ content_id.pdf

15. Zhang J, Wu W, Zhao X, Zhang W. Recommended psychological crisis intervention response to the 2019 novel coronavirus pneumonia outbreak in China: a model of West China Hospital. Precis Clin Med. 2020;3:3-8.

16. Prasad M, Christie JD, Bellamy SL, Rubenfeld GD, Kahn JM. The availability of clinical protocols in US teaching intensive care units. J Crit Care. 2010;25:610-9.

17. Cuijpers P, van Straten A, Andersson G. Internetadministered cognitive behavior therapy for health problems: a systematic review. J Behav Med. 2008;31:169-77.

18. Qiu J, Shen B, Zhao M, Wang Z, Xie B, Xu Y. A nationwide survey of psychological distress among Chinese people in the COVID-19 epidemic: implications and policy recommendations. Gen Psychiatr. 2020;33:e100213. 